\title{
Design and use of biomarkers for the current and future clinical management of brain tumors
}

\author{
"The complex pathophysiology of brain tumors coupled with a need for better \\ markers of prognosis, therapy response and recurrence, underscores the need for \\ clinically useful biomarkers that accurately reflect the entire tumor."
}

\section{KEYWORDS: astrocytoma $\approx$ biomarker $\approx$ glioma $\approx$ immunosignature $\approx$ oligodendroglioma}

Brain tumors encompass a devastating collection of neoplasias, the worst of which are grade IV astrocytomas or glioblastoma (GBM). GBM is the most common malignant primary brain tumor and has dismal survival statistics despite current treatment options, which include surgical resection, radiation and chemotherapy. Some of the major hurdles that contribute to poor clinical outcome relate to the location in the body, difficulty in imaging, diffuse tumor margins, migrating and therapy-resistant cells, a general preference for deep-brain locations and tumor recurrence. Lower grade brain tumors can share these characteristics and they too eventually recur, often as a higher grade tumor. MRI is typically used to follow patients for tumor recurrence; however, it can be difficult to discern between treatment effect and tumor regrowth. Thus, despite the fact that surgical resection carries with it a set of risks, clinical decisions often require a tissue diagnosis. Biopsies are generally thought to reduce this risk somewhat; however, biopsies often provide a less accurate picture of the tumor due to the tumor's inherent heterogeneity. The complex pathophysiology of brain tumors coupled with a need for better markers of prognosis, therapy response and recurrence, underscores the need for clinically useful biomarkers that accurately reflect the entire tumor.

\section{"Any measurable trait in a cell or organism can be postulated to serve as a biomarker of cancer diagnosis, prognosis, therapy response, residual or recurrent disease, or even as a predictor of therapeutic efficacy."}

Alterations in virtually any biological molecule can be used as a biomarker. A PubMed search of 'cancer biomarker' produces a list of almost 200,000 references. Biomarkers are defined by the NIH as having "characteristics that are objectively measured and evaluated as indicators of normal biologic processes, pathogenic processes or pharmacologic responses to a therapeutic intervention" [1]. Any measurable trait in a cell or organism can be postulated to serve as a biomarker of cancer diagnosis, prognosis, therapy response, residual or recurrent disease, or even as a predictor of therapeutic efficacy. As technology has improved to allow highly detailed profiling of diseases such as malignant brain tumors, we have gained commensurately greater understanding of their molecular complexity, and there are now welldefined molecular subtypes of GBMs $[2,3]$. The use of this information for the design of new therapies or markers that might predict diagnosis, prognosis, tumor recurrence or therapy response is just beginning. It is likely that in addition to new markers, we will 'revisit the past' and redefine the utility of genetic alterations that failed to become routinely used for the diagnosis or prognostication of gliomas. For example, early work on biomarkers of malignant brain tumors focused on individual genetic alterations seen in a high percentage of tumors. In most cases these genetic alterations were not robust enough to be used for the routine diagnosis/prognostication of gliomas at the time. Some, such as amplification and/ or mutation of the EGFR gene in GBMs, were postulated as markers of poor prognosis; however, there were conflicting data and this was not accepted as a proven clinical biomarker. We now know that amplification/mutation of the EGFR is a defining trait of one of the GBM subclasses and, as such, it may become a biomarker for that subclass, and for patients that may respond to therapies designed against tumors with that molecular profile. It is likely that these subclasses will help refine drug treatment regiments.

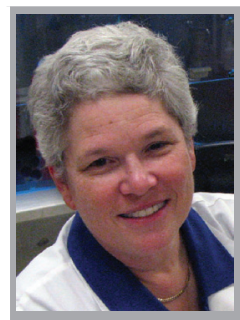

Adrienne C Scheck

Author for correspondence:

Neuro-oncology Research \&

Neurosurgery Research, Barrow

Neurological Institute of St Joseph's

Hospital \& Medical Center, Phoenix,

AZ 85013, USA

Tel.: +1602406 3647

Fax: +16024067172

adrienne.scheck@thebni.ord

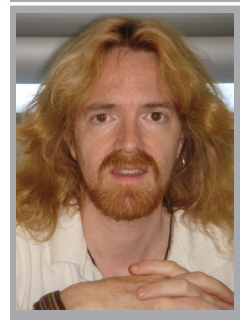

Phillip Stafford

AZ Biodesign, Center for Innovations in Medicine, Arizona State University School of Life Sciences, Tempe, AZ 85287, USA 


\section{Brain tumor biomarkers currently in clinical use}

Allelic loss of chromosomes $1 p / 19 q$

The first biomarker to be universally accepted for clinical use in gliomas is the deletion on the $1 \mathrm{p}$ and $19 \mathrm{q}$ chromosome arms in oligodendrogliomas [4]. This is a diagnostic biomarker for oligodendrogliomas as well as a prognostic marker, as patients whose tumors are codeleted have a better prognosis. Regardless of the method used for the analysis, codeletion (or loss of heterozygosity) of $1 \mathrm{p}$ and $19 \mathrm{q}$ is a very robust biomarker despite the fact that the genes (or precise chromosome region) responsible for this phenotype are unknown. Codeletion of $1 \mathrm{p} / 19 \mathrm{q}$ is now being used as a stratifier in a number of clinical trials.

\section{MGMT promoter methylation}

The second biomarker to gain wide use is predictive of response to chemotherapy. Alkylating agents have been used for the treatment of glioma for over 40 years, beginning with 1,3-bis(2-chloroethyl)1-nitrosourea (BiCNU ${ }^{\circledR}$; Bristol-Myers Squibb Co., NJ, USA) [5], and now focusing mainly on temozolomide (Temodar ${ }^{\circledR}$; Merck \& Co., Inc., NJ, USA). Resistance to BiCNU and temozolomide is mediated, in part, by the DNA repair protein MGMT. Early work demonstrated that MGMT protein levels were not a useful marker for BiCNU response; however, Hegi et al. showed that methylation of the $M G M T$ gene promoter correlated with longer survival in patients treated with temozolomide [6]. Promoter methylation silences gene expression, therefore, tumors with methylated promoters should show a reduction in MGMT mRNA and protein; however, this is not always the case $[7,8]$. This suggests that $M G M T$ promoter methylation may be a surrogate marker for one or more gene changes that result in improved prognosis. Despite the lack of a strong correlation between promoter methylation and gene expression, $M G M T$ promoter methylation is a strong predictor of a better prognosis and is currently being used as a stratifier in some clinical trials.

\section{IDH1/IDH2 mutation}

Another prognostic biomarker is mutation of the genes encoding $I D H 1$ or $I D H 2$. These were discovered by a genome-wide mutational analysis of GBMs [9]. This mutation is thought to be a relatively early event in gliomagenesis and $I D H$ mutations can be found in low-grade astrocytomas, oligodendrogliomas, mixed oligoastrocytomas and secondary GBMs (those that arise from lower grade gliomas). IDH normally decarboxylates isocitrate to $\alpha$-ketoglutarate; however, the mutated form loses this activity and instead converts $\alpha$-ketoglutarate to 2-hydroxyglutarate. $I D H$ mutations are associated with a younger age of tumor onset and are strongly correlated with longer survival regardless of diagnosis [10].

\section{Brain tumor biomarkers under investigation}

The last 5-10 years has seen a tremendous increase in our understanding of the molecular basis of gliomas. The Cancer Genome Atlas and other similar projects have redefined the way we look at gliomas, and options for the design of new biomarkers for the clinical management of gliomas are virtually endless. New imaging modalities, analyses of macromolecules (e.g., DNA, RNA, proteins, lipids and miRNAs) in tumor tissue, blood and cerebral spinal fluid, as well as a greater understanding of what these analyses mean will ultimately lead to better clinical outcomes. However, it must be noted that the utility of any biomarker or combination of biomarkers is only as good as the sample on which the test is performed. The use of tumor tissue for biomarker analysis can be complicated by the inherent heterogeneity of these tumors. Removing a brain tumor in toto can be more complex than removing tumors from other parts of the body and many hospitals prefer to use biopsies for diagnosis. For these reasons, a number of investigators are using blood, serum and/or cerebral spinal fluid to identify biomarkers of gliomas including proteins, metabolites, microvesicle RNA and miRNAs [11-17]. These studies have met with variable success and there can be concerns regarding their sensitivity and specificity. The need for robust serum biomarkers that circumvent these issues has been emphasized by many in the brain tumor field.

\section{"Antibodies make excellent biomarkers because they are stable in serum, have high specificity and affinity for their cognate antigen, are abundant and enable retrospective studies."}

To this end, Stafford and coworkers have invented a microarray-based technology that they term 'immunosignaturing' $[18,19]$. They can capture the dynamics of circulating antibodies and determine the class (and in some cases, subclass) of disease often before symptoms manifest. Antibodies make excellent biomarkers because they are stable in serum, have high specificity and affinity for their cognate antigen, are abundant 
and enable retrospective studies. The technique is simple, inexpensive and highly sensitive to changes in antibody repertoire. Immune surveillance occurs continuously and is quite sensitive to modifications in proteins, whether it is introduction of cancer-specific chimeras such as $\mathrm{BCR}-\mathrm{ABL}$ or modification of existing proteins such as MUC1, which undergoes a modification of its glycosylation status in cancer patients. It has been demonstrated that cancer cells elicit a detectable humoral immune response [20]. Since we have no way of knowing the precise antigens that may elicit an immune response in gliomas, much less those that convey information about pathology or molecular subtypes, Stafford et al. created a single-use microarray composed of 10,000 different random peptides in order to capture the pattern of antibody binding rather than looking for single antibodies to a cancer antigen [18,19]. As opposed to phage display or other panning techniques, these microarrays retain information about partial binding. By changing the length of time that an antibody is given to interact with these random peptides, or by changing the energy of the

\section{References}

Papers of special note have been highlighted as: " of interest

1 Farias-Eisner G, Bank AM, Hwang BY et al. Glioblastoma biomarkers from bench to bedside: advances and challenges. Br. J. Neurosurg. 26(2), 189-194 (2012).

2 Ducray F, Idbaih A, Wang XW, Cheneau C, Labussiere M, Sanson M. Predictive and prognostic factors for gliomas. Expert Rev. Anticancer Ther. 11, 781-789 (2011).

3 Brennan C. Genomic profiles of glioma. Curr. Neurol. Neurosci. Rep. 11, 291-297 (2011).

- Describes the molecular subtypes identified in glioblastomas.

4 Cairncross JG, Ueki K, Zlatescu MC et al. Specific genetic predictors of chemotherapeutic response and survival in patients with anaplastic oligodendrogliomas. J. Natl Cancer Inst. 90, 1473-1479 (1998).

- $\quad$ First paper that described a biomarker for brain tumors, which is still used clinically.

5 Walker MD, Hurwitz BS. BCNU (1,3-bis(2chloroethyl)-1-nitrosourea; NSC-409962) in the treatment of malignant brain tumor a preliminary report. Cancer Chemother. Rep. 54, 263-271 (1970).

6 Hegi ME, Diserens AC, Godard S et al. Clinical trial substantiates the predictive value of $O-6$-methylguanine-DNA methyltransferase promoter methylation in glioblastoma patients treated with

system during the incubation step, a great deal of thermodynamic and kinetic information can be extracted. In this way they classified blinded glioma patient samples into precise groups that matched the tumor pathology and a molecular biomarker, MGMT promoter methylation [12]. In the future, this technique could be used to follow the efficacy of treatment and distinguish tumor recurrence from treatment effect. This technique is also less susceptible to false-positives and -negatives due to tumor heterogeneity since the body creates its own response to the tumor. The future use of biomarkers such as this will improve the clinical management of malignant gliomas.

\section{Financial \& competing interests disclosure}

The authors have no relevant affiliations or financial involvement with any organization or entity with a financial interest in or financial conflict with the subject matter or materials discussed in the manuscript. This includes employment, consultancies, honoraria, stock ownership or options, expert testimony, grants or patents received or pending, or royalties.

No writing assistance was utilized in the production of this manuscript.

temozolomide. Clin. Cancer Res. 10, 1871-1874 (2004).

7 Christmann M, Nagel G, Horn S et al. MGMT activity, promoter methylation and immunohistochemistry of pre-treatment and recurrent malignant gliomas: a comparative study on astrocytoma and glioblastoma. Int. J. Cancer 127(9), 2106-2118 (2010).

8 Karayan-Tapon L, Quillien V, Guilhot J et al. Prognostic value of O6-methylguanine-DNA methyltransferase status in glioblastoma patients, assessed by five different methods. J. Neurooncol. 97, 311-322 (2010).

9 Yan H, Parsons DW, Jin Get al. IDH1 and IDH2 mutations in gliomas. N. Engl. J. Med. 360, 765-773 (2009).

10 Guo C, Pirozzi CJ, Lopez GY, Yan H. Isocitrate dehydrogenase mutations in gliomas: mechanisms, biomarkers and therapeutic target. Curr. Opin Neurol. 24, 648-652 (2011).

11 Balana C, Carrato C, Ramirez JL et al. Tumour and serum MGMT promoter methylation and protein expression in glioblastoma patients. Clin. Transl. Oncol. 13, 677-685 (2011).

12 Hughes A, Scheck AC, Coons SW, Stafford P, Johnston SA. Immunosignaturing can detect products from molecular markers in brain cancer. PLoS ONE (2012) (In Press).

13 Lavon I, Refael M, Zelikovitch B, Shalom E, Siegal T. Serum DNA can define tumorspecific genetic and epigenetic markers in gliomas of various grades. Neuro. Oncol. 12, 173-180 (2010).
14 Ludwig N, Keller A, Comtesse N et al. Pattern of serum autoantibodies allows accurate distinction between a tumor and pathologies of the same organ. Clin. Cancer Res. 14, 4767-4774 (2008).

15 Roth P, Wischhusen J, Happold C et al. A specific miRNA signature in the peripheral blood of glioblastoma patients. J. Neurochem. 118, 449-457 (2011).

16 Somasundaram K, Nijaguna MB, Kumar DM. Serum proteomics of glioma: methods and applications. Expert Rev. Mol. Diagn. 9, 695-707 (2009).

$17 \mathrm{Xu}$ BJ, An QA, Srinivasa GS et al. Identification of blood protein biomarkers that aid in the clinical assessment of patients with malignant glioma. Int. J. Oncol. 40(6), 1995-2003 (2012).

18 Stafford P, Halperin R, Legutki JB, Magee DM, Galgiani J, Johnston SA. Physical characterization of the 'immunosignaturing effect'. Mol. Cell. Proteomics 11(4), M111.011593 (2012).

19 Stafford P, Johnston S. Microarray technology displays the complexities of the humoral immune response. Expert Rev. Mol. Diagn. 11, 5-8 (2011).

- Describes the immunosignaturing technology.

20 Mintz PJ, Kim J, Do KA et al. Fingerprinting the circulating repertoire of antibodies from cancer patients. Nat. Biotechnol. 21, 57-63 (2003). 